\title{
Fluctuations in an Equilibrium Convective Ensemble. Part I: Theoretical Formulation
}

\author{
George C. Craig \\ DLR Institut für Physik der Atmosphäre, Oberpfaffenhofen, Germany \\ Brenda G. COHEN \\ Department of Meteorology, University of Reading, Reading, United Kingdom
}

(Manuscript received 22 March 2005, in final form 14 October 2005)

\begin{abstract}
To provide a theoretical basis for stochastic parameterization of cumulus convection, the equilibrium fluctuations of a field of cumulus clouds under homogeneous large-scale forcing are derived statistically, using the Gibbs canonical ensemble from statistical mechanics. In the limit of noninteracting convective cells, the statistics of these convective fluctuations can be written in terms of the large-scale, externally constrained properties of the system. Using this framework, the probability density function of individual cloud mass fluxes is shown to be exponential. An analytical expression for the distribution function of total mass flux over a region of given size is also derived, and the variance of this distribution is found to be inversely related to the mean number of clouds in the ensemble. In a companion paper, these theoretical predictions are tested against cloud resolving model data.
\end{abstract}

\section{Introduction}

The parameterization of cumulus convection in numerical models of the atmosphere is usually based upon a local equilibrium hypothesis, where it is assumed that the average properties of the (unresolved) convection within each grid box can be determined solely in terms of large-scale (resolved) conditions. In particular, the heating, moistening, and momentum sources of the convection are functions of the temperature, moisture content, and other model variables in the relevant column (Ooyama 1971; Arakawa and Schubert 1974; Gregory and Miller 1989; Tiedke 1989; Emanuel 1991). This assumption can only be exactly true in the limit of averaging over an infinite number of clouds. For a region of finite size, the average convective properties will fluctuate around the equilibrium average, depending on how many clouds happen to be in the region at any given moment.

Deviations from equilibrium are important for at least three reasons. First, subgrid-scale convective fluc-

Corresponding author address: George C. Craig, DLR Oberpfaffenhofen, Institut für Physik der Atmosphäre, Münchner Strasse 20, D-82234 Wessling, Germany.

E-mail: george.craig@dlr.de tuations are a significant source of variability for the larger-scale climate (Lin and Neelin 2000). Second, quantitative precipitation forecasting often requires predictions for very small regions (e.g., river catchments), which are only probabilistically related to the larger-scale flow that is observed and forecast (Fritsch et al. 1998). Finally, convective variability is a significant source of model error in deterministic numerical weather prediction models and can influence the spread of ensemble forecasts (Buizza et al. 1999; Palmer 2001).

It is therefore an important task to characterize convective variability. In particular, one would hope to predict the statistics of the convective fluctuations in terms of the same large-scale properties that determine the mean behavior. This paper attempts to provide such a characterization using standard methods from statistical physics for describing fluctuations about an equilibrium. The second part of this study will describe tests of the theoretical predictions using a cloud resolving model.

Before attempting an equilibrium fluctuation theory, it is important to distinguish the convective variability due to local fluctuations in an equilibrium situation from variability that occurs when the convection is not in equilibrium at all. If the forcing for convection is varying on time and space scales similar to convective 
motions themselves, the convection will not have the opportunity to reach equilibrium, and it will not be possible to distinguish the variability of the forcing from the intrinsic variability of the convection. For equilibrium, the forcing must be sufficiently slowly varying in space that it can be considered uniform over a region large enough to contain many clouds. It must be sufficiently slowly varying in time that the convection has time to adjust to changes in its environment. In fact, these two requirements are closely related, since it appears that the adjustment time is linearly proportional to the cloud spacing (Cohen and Craig 2004). In this paper, only the equilibrium situation will be considered; attention will be focused on the spatial variability that occurs within a finite-sized region such as the grid box of a numerical model. Cohen and Craig (2004) suggest that for reasonable variations in convective forcing, the convective ensemble is in a linear response regime, in which case it may be possible to generalize the results here to at least some nonequilibrium situations.

The statistical characterization of small-scale variability in turbulent flows can be a very complex task, even for the simple case of homogeneous turbulence (Lesieur 1997). But moist atmospheric convection has a useful simplification that is not found in most turbulent systems. The fraction of the atmosphere occupied by active convective motions is small (Bjerknes 1938; Bretherton 1988; Cotton and Anthes 1989) and, in the absence of convective organization, it may be a reasonable hypothesis to regard convection as composed of isolated entities that interact with each other only through the mean flow. This is analogous to the "ideal" assumption for an ideal gas, where the gas is presumed to be composed of particles whose statistical properties are determined by the energy and particle number of the whole system, and not influenced significantly by the details of how the molecules interact locally in collisions (Landau and Lifshitz 1968). This idealized model of convection will lead to a statistical description, along the lines of the phenomenological descriptions of Cho (1978) or Randall and Huffman (1980), but based on a small number of physically motivated assumptions. The underlying physical model suggests that the predictions will apply best to situations of weakly forced, unorganized convection, and provides estimates of the range of spatial scales where the predictions should apply.

Section 2 of the paper discusses the assumptions underlying the development in more detail, and attempts to identify the physical parameters that will form the basis of the theory. The main results are derived in sections 3 and 4, which present probability density func- tions for the mass flux of a single cloud and the total mass flux over a region of given size, respectively. Section 5 presents a discussion of the implications of the results, and methods for testing the predictions.

\section{Assumptions and constraints}

In this section, the assumptions underlying the statistical derivations of the subsequent sections are discussed. An attempt will be made to provide physical motivation for each statement, but these cannot be regarded as compelling arguments. Indeed, other selfconsistent theories could be constructed using different sets of assumptions. The particular choices made here can only be assessed by testing the conclusions derived from them.

\section{a. Large-scale constraints on the convective ensemble}

We take, as a starting point, a conventional mass flux picture of the relationship between an ensemble of convective clouds and its environment (Ooyama 1971; Gregory 1997). The atmosphere is rendered unstable to moist convection on the large-scale through some combination of radiative cooling, large-scale ascent that produces adiabatic cooling, and surface fluxes of heat and moisture. The convection, consisting of moist updrafts and downdrafts, acts to reduce the instability by a net transport of mass upward. Some or all of this mass descends between the convective updrafts, warming the atmosphere by adiabatic compression, and thus tending to restore stability. If the area occupied by the convective updrafts and downdrafts is small and reevaporation of precipitation is ignored, it can be shown that the net convective mass flux at equilibrium is constrained by the need to produce subsidence warming equal to the large-scale cooling that forces the convection (Gregory and Miller 1989). We therefore assume that the mean convective mass flux over the area of interest at some arbitrary height, $\langle M\rangle$ (in units of $\mathrm{kg} \mathrm{s}^{-1}$ ) is constrained by the large-scale flow. The angle brackets denote an ensemble mean over all possible realizations of the convection consistent with the large-scale forcing conditions. The calculation of the mean convective mass flux at cloud-base level is a standard part of mass flux cumulus parameterizations.

The variability of equilibrium convection results from the fact that the mean mass flux, $\langle M\rangle$, is divided up among a number of finite-sized clouds. A given region may contain various numbers of clouds of various sizes, although the total mass flux must approach the average, $\langle M\rangle$, if the region is very large. It is thus necessary to 
characterize the degree to which the convection is broken up, with some measure of the typical number of clouds or their typical size. The size of a convective cloud is likely to be determined by properties local to that cloud-the initial perturbation that triggers it, and the mixing processes that accompany its development. We therefore take as a second fundamental parameter describing the convective ensemble the mean mass flux per cloud, $\langle m\rangle\left(\mathrm{kg} \mathrm{s}^{-1}\right)$. Some evidence for this is provided by the cumulus ensemble simulations of Robe and Emanuel (1996) that show the convective vertical velocity to be almost independent of the large-scale forcing and mean convective mass flux. This invariance of the mass flux to the forcing has also been noted by Cohen (2001). A potential alternative to using the instantaneous cloud mass flux would be to assume a constant total mass flux integrated over the lifetime of a cloud. This would yield the same results if the mass flux were constant over the life of the cloud, or if all clouds followed the same well-defined life cycle. In principle, the time-integrated mass flux is more appealing since it is independent of the time evolution of the cloud; however, it is much harder to calculate in practice, and therefore is not used here.

\section{b. Scale separation}

We assume that a scale separation exists in time and space between variations in the large-scale forcing and in the convective ensemble. In particular, it is assumed that it is possible to define a region that is small enough in space that the forcing of convection does not vary significantly over the region, and yet large enough to contain many clouds (Yanai et al. 1973; Arakawa and Schubert 1974). This assumption implies that the average quantities, $\langle M\rangle$ and $\langle m\rangle$, which characterize the convective ensemble, are well defined. We further assume that the forcing varies sufficiently slowly in time that the convection has time to reach and remain close to equilibrium. This implies that $\langle M\rangle$ and $\langle m\rangle$ can be determined solely in terms of the large-scale forcing.

\section{c. Weak interactions}

If the convective clouds are sufficiently separated it may be reasonable to ignore the interactions of a cloud with its immediate neighbors. The cloud will feel only an aggregate change to its environment resulting from the effects of all the other clouds. In other words, the clouds will interact only through their effects on the large-scale state of the atmosphere. Consistent with this approximation, the extent of individual clouds is ignored and the clouds are assumed to be pointlike. This is reasonable only if attention is restricted to regions

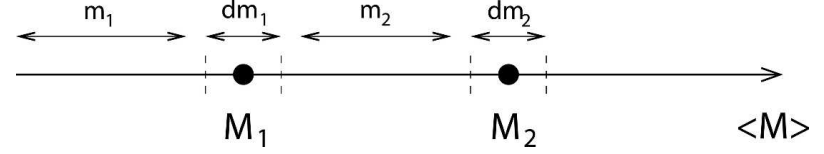

FIG. 1. Division of a mass flux interval into two clouds with mass fluxes $M_{1}$ and $M_{2}$.

large compared to the cloud size. The noninteraction idealization implies that any aspect of the convective variability can be described in terms of the mean properties of the ensemble; that is, $\langle M\rangle$ and $\langle m\rangle$. For example, the mean number of clouds in the area of interest is given by $\langle N\rangle=\langle M\rangle /\langle m\rangle$.

\section{d. Equal a priori probabilities}

A final assumption, key to all of equilibrium statistical mechanics, is the postulate of equal a priori probabilities. We assume that clouds are equally likely to occur in any location and with any mass flux, subject only to the constraints described above, namely that the mean mass flux, $\langle M\rangle$, and the mean mass flux per cloud, $\langle m\rangle$, are as required by the large-scale forcing. This assumption cannot be justified, except by its success in the statistical description of many natural phenomena.

\section{Probability distribution of mass flux per cloud}

In this section, the distribution of individual cloud mass fluxes is described in terms of the mean mass flux per cloud, $\langle m\rangle$, which is assumed to be independent of the forcing. Since we assume that the clouds are noninteracting and thus independent of their neighbors, we do not need to consider the spatial locations of the clouds in this calculation. The derivation here is formulated in terms of stochastic processes, but the resulting exponential probability distribution of cloud mass fluxes is the well-known Boltzmann distribution, obtained from the statistical mechanics of the canonical ensemble (see the appendix).

To compute how the mass flux in a given region is distributed among the individual clouds, we consider the following construction. The total mass flux is represented as a line, with the mass fluxes of the individual clouds corresponding to intervals on the line (Fig. 1). For example, the distance from the origin to the dividing point $M_{1}$ is the mass flux of one cloud, and the distance from $M_{1}$ to the next point $M_{2}$ is the mass flux of a second cloud. The fixed mean mass flux per cloud can be written inversely as a constraint on the mean number of clouds per unit mass flux:

$$
\lambda=\frac{\langle N\rangle}{\langle M\rangle}=\frac{1}{\langle m\rangle},
$$


or equivalently the number of dividing points per unit length. The number of clouds contributing to a given mass flux $M$ is a random variable, whose distribution, $p_{M}(n)$ is given by the probability that the number of dividing points that fall into an interval of length $M$, $N((0, M])$, is equal to $n$ (the round bracket indicates that the endpoint 0 is not included in the interval, while the square bracket indicates that the endpoint $M$ is included). Since the mass fluxes of the individual clouds are assumed to be uncorrelated, the distribution, $p_{M}(n)$, can be described by the Poisson point process:

$$
\begin{aligned}
p_{M}(n) \equiv \operatorname{Prob}\{N((0, M])=n\} & =\frac{(\lambda M)^{n} e^{-\lambda M}}{n !} \text { for } \\
n & =0,1, \ldots,
\end{aligned}
$$

with rate parameter $\lambda=(1 /\langle m\rangle)$.

From Eq. (2) it can be shown that the distribution of interval lengths between points is governed by an exponential distribution, and hence that the mass flux of individual clouds is exponentially distributed. This result will be demonstrated here for a mass flux interval containing exactly two clouds, but can easily be extended to a longer interval containing any number of clouds.

Consider an interval of mass flux, subdivided into two clouds with individual mass fluxes in the range $m_{1}$ to $m_{1}+d m_{1}$ and $m_{2}$ to $m_{2}+d m_{2}$, as shown in Fig. 1 . The joint probability density function for $m_{1}$ and $m_{2}$ can be written as a product of the probabilities of exactly one dividing point $\left(M_{1}\right.$ or $M_{2}$, respectively) occurring in each of the intervals $\left(m_{1}, m_{1}+d m_{1}\right]$ and $\left(m_{2}, m_{2}+\right.$ $d m_{2}$ ] (Taylor and Karlin 1994), that is,

$$
\begin{aligned}
p_{M_{1}, M_{2}}\left(m_{1}, m_{2}\right) d m_{1} d m_{2}=\operatorname{Prob}\left\{N \left(\left(\left[0, m_{1}\right]\right)\right.\right. & =0\} \\
\times \operatorname{Prob}\left\{N\left(\left(m_{1}, m_{1}+d m_{1}\right]\right)\right. & =1\} \\
\times \operatorname{Prob}\left\{N\left(\left(m_{1}+d m_{1}, m_{1}+d m_{1}+m_{2}\right]\right)\right. & =0\} \\
\times \operatorname{Prob}\left\{N\left(\left(m_{1}+d m_{1}+m_{2}, m_{1}+d m_{1}+m_{2}+d m_{2}\right]\right)\right. & =1\} .
\end{aligned}
$$

Using Eq. (2), this can be rewritten as

$$
\begin{aligned}
p_{M_{1}, M_{2}}\left(m_{1}, m_{2}\right) d m_{1} d m_{2}= & \left(e^{-\lambda m_{1}}\right)\left(e^{-\lambda m_{2}}\right)\left(\lambda d m_{1} e^{-\lambda d m_{1}}\right) \\
& \times\left(\lambda d m_{2} e^{-\lambda d m_{2}}\right) .
\end{aligned}
$$

Upon dividing both sides of (4) by $\left(d m_{1}\right)\left(d m_{2}\right)$, and taking the limit as $d m_{1}, d m_{2} \rightarrow 0$, it is found that

$$
p_{M_{1}, M_{2}}\left(m_{1}, m_{2}\right)=\left(\lambda e^{-\lambda m_{1}}\right)\left(\lambda e^{-\lambda m_{2}}\right) \text {. }
$$

Finally, using the fact that individual clouds are independent, the probability distribution of mass flux per cloud can be written in general form as

$$
p(m) d m=\frac{1}{\langle m\rangle} e^{-m /\langle m\rangle} d m .
$$

This result is analogous to the Boltzmann distribution, with $\langle m\rangle$ playing the role of temperature.

Since the mean number of clouds contributing to the total mass flux is $\langle N\rangle$, the average number of clouds in the region that possess a mass flux between $m$ and $m+$ $d m$ is

$$
d \bar{n}(m)=\langle N\rangle p(m) d m=\frac{\langle N\rangle}{\langle m\rangle} e^{-m /\langle m\rangle} d m .
$$

This final equation is in a form suitable for verification against observations or numerical model data.

\section{Probability distribution of total mass flux}

\section{a. Probability density function}

The probability distribution of the total mass flux of the cloud ensemble in a given region of finite size, $p(M)$, can be derived by combining the distribution of individual mass fluxes from (6) with the distribution of cloud number in the region. The weak interaction assumption implies that the locations of the clouds are uncorrelated, and thus the number of clouds is governed by a Poisson distribution:

$$
p_{N}(n)=\frac{\langle N\rangle^{n} e^{-\langle N\rangle}}{n !} \text { for } n=0,1, \ldots
$$

It is most straightforward to consider firstly the situation where the number of clouds in the ensemble is fixed to be exactly $N$. In this situation, the probability density function for the total mass flux of the ensemble, $p^{(N)}(M)$, can be calculated as an $N$-fold convolution over $p(m)$ (Taylor and Karlin 1994); that is,

$$
p^{(N)}(M)=\int_{0}^{M} p^{(N-1)}(M-u) p(u) d u .
$$

This gives, on recursive substitution of (6) into (9)

$$
p^{(N)}(M)=\frac{1}{(N-1) !}\left(\frac{1}{\langle m\rangle}\right)^{N} M^{N-1} e^{-M /\langle m\rangle} .
$$

Next, in order to relax the assumption of fixed $N$, we sum over (10) for all values of $N=n$, weighted by the probability of there being exactly $n$ clouds, $p_{N}(n)$, from (8); that is,

$$
p(M)= \begin{cases}\sum_{n=1}^{\infty} p^{(n)}(M) p_{N}(n) \text { for } \quad M \geq 1 \\ p_{N}(n=0)=e^{-\langle N\rangle} \text { for } \quad M=0 .\end{cases}
$$

Here, $p(M)$ has a discrete component, corresponding to the case when there are no clouds in the region; that is, $n=0$ and $M=0$, and a continuous distribution when $M$ $\geq 1$, or equivalently $n \geq 1$. 

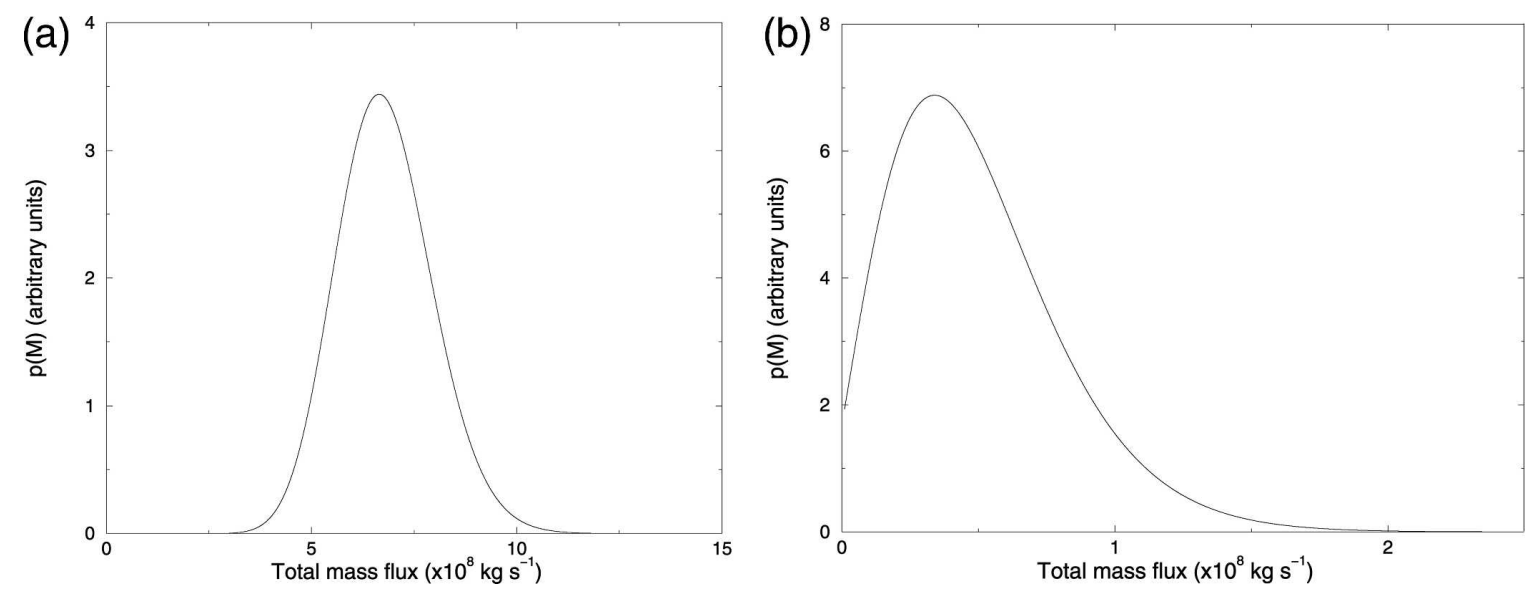

FIG. 2. Theoretical probability distributions of total mass flux, $p(M)$ : (a) $\langle N\rangle=68$; (b) $\langle N\rangle=5$; $\langle m\rangle=10^{7} \mathrm{~kg} \mathrm{~s}^{-1}$ for both curves.

For $n \geq 1$, then, we find by substituting Eqs. (10) and (8) into (11):

$$
p(M)=\frac{\langle N\rangle}{\langle m\rangle} e^{-\langle N\rangle} e^{-M /\langle m\rangle} \sum_{n=1}^{\infty} \frac{\left(\frac{\langle N\rangle}{\langle m\rangle} M\right)^{n-1}}{n !(n-1) !} .
$$

The summation in Eq. (12) is of standard form, and can be written as

$$
\sum_{n=1}^{\infty} \frac{x^{n-1}}{n !(n-1) !} \equiv \frac{I_{1}(2 \sqrt{x})}{\sqrt{x}}
$$

where $I_{1}(x)$ is the modified Bessel function of order 1.

Hence, for our cloud system, the probability distribution of total mass flux is given by

$$
p(M)=\left(\frac{\langle N\rangle}{\langle m\rangle}\right)^{1 / 2} e^{-\langle N\rangle} M^{-1 / 2} e^{-M /\langle m\rangle} I_{1}\left(2 \sqrt{\frac{\langle N\rangle}{\langle m\rangle} M}\right) .
$$

This expression is valid for a convective ensemble containing any number of clouds (greater than zero). As discussed further in section 5 , a version of this formula has been presented in a related context by RodriguezIturbe et al. (1987).

The form of this distribution is not easily recognizable, and so the shape of $p(M)$ is plotted in Figs. 2a,b for two different values of $\langle N\rangle$, corresponding to a small region $(\langle N\rangle=5)$ and a region containing a large number of clouds $(\langle N\rangle=68)$. In accordance with the central limit theorem, the distribution becomes more Gaussian as $\langle N\rangle$ increases, and the variance decreases correspondingly. The shape is reminiscent of a Poisson distribution, which would have been the solution if all clouds were constrained to have the same fixed mass flux.

\section{b. Fluctuations in total mass flux}

The variance of the total convective mass flux in a region can be calculated from the distribution calculated in the previous section; however, it can also be obtained directly from the initial assumptions in a way that gives some physical insight into the factors controlling the variability. The total mass flux of an arbitrary $n$-cloud system can be written as

$$
M(n)=\sum_{i=1}^{n} m_{i}
$$

where $m_{i}$ is the mass flux of an individual cloud. Here, the probability distribution of each $m_{i}$ is identical [given by Eq. (6)], and the clouds are assumed to evolve independently of one another. Now, defining the probability distribution of the number of particles in the general case as $p(n)$, the theory of random sums can be invoked to provide predictions for the mean and variance of $M(n)$ in terms of $\langle m\rangle$ and $\langle N\rangle$ [the derivation is straightforward and is given in Taylor and Karlin (1994)]. Specifically, for the assumed random, Poisson distribution of cloud number [Eq. (8)], the predictions are

$$
\langle M\rangle=\langle N\rangle\langle m\rangle,
$$

in agreement with Eq. (1), and

$$
\left\langle(\delta M)^{2}\right\rangle=2\langle N\rangle\langle m\rangle^{2} .
$$

Equation (17) can be normalized by the mean mass flux, to give

$$
\frac{\left\langle(\delta M)^{2}\right\rangle}{\langle M\rangle^{2}}=\frac{2}{\langle N\rangle} .
$$

This final expression is simply a mathematical statement of the intuitive result that the variance scales in- 
versely with the number of clouds in the ensemble. However, it is exactly 2 times the variance that would have been obtained if all clouds had the same mass flux, showing that the random positions of clouds and their random mass flux make equal contributions to the total mass flux variance.

\section{Discussion}

\section{a. Meaning of parameters}

Expressions have been derived in this paper for the distribution of mass flux carried by individual clouds and of the total convective mass flux over a region of finite size, for a convecting atmosphere in equilibrium with the large-scale forcing. Two parameters are required, the mean convective mass flux, $\langle M\rangle$, and the mean mass flux per cloud, $\langle m\rangle$. As noted previously, $\langle M\rangle$ is determined by the requirement that the convection balance the large-scale forcing when averaged over a large region, and is provided, for example, by the closure in a mass flux convection scheme. The only new parameter that would be required to introduce this description of variability in a parameterization would be $\langle m\rangle$.

The second parameter, $\langle m\rangle$, is not necessarily a function of the large-scale forcing. Some results (Robe and Emanuel 1996; Cohen 2001) suggest that it may even be independent of the forcing; that is, that the response to a change in forcing is to change the number of clouds, rather than the properties of the individual clouds. Consistent with this $\mathrm{Xu}$ et al. (2005) found, in a comparison of satellite-derived cloud properties between El Niño and La Niña regimes, that the frequency of cloud occurrence changed; while many other statistics, such as the frequency distribution of cloud optical depths, did not change. This insensitivity to large-scale forcing would indicate that the mass flux of an individual cloud is related to the initial perturbation that triggers it and the entrainment processes that modify its mass flux over time. For example it could be expected that the mass fluxes of tropical and midlatitude clouds would be different (Xu and Randall 2001). At present there can be little certainty about how $\langle m\rangle$ is determined since the crucial processes are not observed in sufficient detail, nor resolved in most numerical simulations. However, the key role that this parameter plays in determining the overall convective variability makes it an important candidate for future research.

\section{b. Interpretation of mass flux variability}

The variability of convective mass flux in a given region will be small if the number of clouds is large; that is, if the size of the region is large in comparison to the typical cloud separation distance. Thus, a necessary condition for convection in a finite region, such as a grid box in a numerical model, to be in equilibrium with the average forcing over that region is that the region size $\Delta x$ be larger than the mean cloud spacing, $L=$ $(A /\langle N\rangle)^{1 / 2}=(\langle m\rangle A /\langle M\rangle)^{1 / 2}$, where $A$ is the size of area containing on average $\langle N\rangle$ clouds. It is interesting to note that, since $\langle m\rangle$ appears to be independent of the forcing, $L$ is inversely related to $\langle M\rangle$. Where convection is strongly forced, there are more clouds to average over and less convective variability in a region of a given size. An equilibrium convective parameterization will be most accurate where it is most needed.

An estimate of the magnitude of convective variability can easily be obtained from typical values of $\langle m\rangle$ and $\langle M\rangle$. For a purely radiative-convective equilibrium, latent heat release must balance radiative cooling $S$. If the rate of latent heating is estimated crudely as the convective mass flux multiplied by a typical water vapor mass mixing ratio, $q$, we obtain

$$
l_{v} q \frac{\langle M\rangle}{A}=S,
$$

where $l_{v}$ is the latent heat of condensation. Values of $S=250 \mathrm{~W} \mathrm{~m}^{-2}$ and $q=10 \mathrm{~g} \mathrm{~kg}^{-1}$ give $\langle M\rangle / A=10^{-2}$ $\mathrm{kg} \mathrm{s}^{-1} \mathrm{~m}^{-2}$. Estimating $\langle m\rangle$ for a deep convective cloud with a $10 \mathrm{~m} \mathrm{~s}^{-1}$ vertical velocity and a size of $1 \mathrm{~km}^{2}$ gives $\langle m\rangle=10^{7} \mathrm{~kg} \mathrm{~s}^{-1}$. The characteristic cloud spacing is then approximately $L=30 \mathrm{~km}$. The normalized standard deviation of convective variability in a square region with side $\Delta x$ is [from Eq. (18)]

$$
\frac{\delta M}{M}=2^{1 / 2} \frac{L}{\Delta x},
$$

which gives a value of only 0.4 if $\Delta x=100 \mathrm{~km}$, a typical resolution for a global model, but 2.1 for $\Delta x=20 \mathrm{~km}$, a typical mesoscale model resolution. In regions of substantial mean ascent the convective forcing will be larger and the variability smaller: for example an increase in forcing by a factor of 4 will reduce the variability to half the radiative-convective value. For comparison, in the stochastic parameterization scheme of Buizza et al. (1999), the optimum value of random perturbation applied to the equilibrium tendency was found to be in the range 0.5 to 1.5 , for a $10^{\circ} \times 10^{\circ}$ region. This introduces a magnitude of variability that is larger than would be predicted from the statistical theory, but is perhaps reasonable for situations in which convective organization is prevalent. 


\section{c. Experimental validation}

A comparison of the distributions found in this work with observations is essential, but difficult for two reasons. First, sufficient data is needed to define the mass flux distribution, especially for the largest clouds where the various distribution functions that have been proposed (discussed below) show their greatest differences. This is particularly difficult since, as indicated above, the parameters of the distribution function depend upon the forcing of convection, so that data must be stratified or rescaled according to the environmental conditions. Second, the constrained variable in the theory, convective mass flux, is difficult to observe directly. Comparisons of the predictions for mass flux to observations of other quantities such as radar reflectivity or visible cloud size are unlikely to be quantitative, and may even show qualitative differences due to the complex interactions of dynamics and microphysics that produce the observed patterns. The existing observations will be reviewed briefly below. An alternative way of testing the theory is to use simulated data from a cloud resolving model; this is the subject of Part II of this work (Cohen and Craig 2006, hereafter Part II).

Direct observations of the mass flux of cumulus clouds have been made by Jorgensen and Lemone (1989), although their data was not sufficiently representative to determine if the distribution was exponential. Exponential distributions of cloud size using imagery from aircraft or satellite have been reported by a number of authors (Plank 1969; Hozumi et al. 1982; Astin and Latter 1998), while in other studies that are based on radar reflectivity, data have been fitted to a lognormal distribution (Lopez 1978; Lopez et al. 1984). In this case, however, the difference between lognormal and exponential distributions may be unclear, since reflectivity will not reflect the population of small clouds that contain few precipitation particles. Finally, many analyses, particularly more recent work, have shown power-law distributions of cloud size, usually with a scale break (e.g., Cahalan and Joseph 1989; Sengupta et al. 1990; Nair et al. 1998). This may result from nonideal behavior in the mass flux, but may also result from the different processes that affect cloud size. In particular, clouds persist and spread even after the convective updraft weakens, and are thus more likely than the updrafts themselves to interact and merge with their neighbors. This merging may lead to percolation and the emergence of power-law scaling (Stauffer and Ahorony 1992). It is interesting that some of the "young" cloud fields studied by Sengupta et al. (1990) and Nair et al. (1998) show distributions that are not far from exponential, while the more mature cloud fields are clearly fit by a power law. It may be that the visible cloud size more closely represents the mass flux of a cloud early in its life cycle.

The spatial distribution of clouds, assumed to be random in this paper, and the corresponding distribution function for mass flux can also be compared to observations. The results here are inconclusive since random distributions (Nair et al. 1998), spatial regularity (Ramirez et al. 1990; Nair et al. 1998), and clustering of clouds (Cahalan and Joseph 1989; Sengupta et al. 1990) have all been observed. The behavior is presumably sensitive to the environment where the clouds are forming, but it is not clear what specific factors are responsible for a particular behavior.

A better proxy for mass flux than cloud area might be precipitation, which is directly related to the columnintegrated latent heating. The spatial distribution of precipitation has been extensively studied in the hydrological literature (Gupta and Waymire 1979; Kavvas and Delleur 1981; Eagleson 1978), and one study in particular is especially relevant to the work presented here. Rodriguez-Iturbe et al. (1987) obtained a theoretical cumulative rainfall probability distribution exactly analogous to Eq. (14), and compared this prediction against monthly precipitation data from Denver, Colorado, collected over a time span of $28 \mathrm{yr}$. The agreement was poor. However, this comparison assumed constant values of $\langle M\rangle$ and $\langle N\rangle$ throughout the observing periods, whereas in reality these quantities vary significantly as a function of large-scale forcing conditions. To provide a quantitative validation of the theoretical distribution, therefore, would require a more sophisticated approach, such as rescaling the data by the changing mean total mass flux value, $\langle M\rangle$ (assuming a constant value of $\langle m\rangle)$, to produce a single probability distribution. It would seem useful to revisit this analysis, incorporating the influence of the environment, as indicated above.

\section{APPENDIX}

\section{The Analogy with the Ideal Gas}

It is interesting to compare the conceptual model of convective activity presented in this work with the ideal gas model, which to some extent motivated it. An ideal gas is assumed to be a system of particles that satisfy the following assumptions. First, there is a scale separation that allows one to consider a large number of particles with well-defined averages. Additionally, the gas system is assumed to be in equilibrium, and the particles are assumed to be weakly interacting, so that their statistics are functions of the mean properties of the system. Finally, all states of the system consistent with 
these assumptions are assumed to be equally probable. Real gases tend to show ideal behavior only in the lowdensity limit; when particles are closely packed, interactions become significant and nonideal behavior is observed.

The large-scale constraints that the system of particles must satisfy are to some extent analogous to those of the convective system. In particular, the requirement that energy, $E$, is conserved (or conserved in the mean for a system in contact with a large reservoir of heat with fixed temperature) corresponds to the requirement that convective mass flux, $\langle M\rangle$, is constrained in the mean. This is equivalent to regarding the system (convection in a given region) as a small part of a much larger system (an infinite convection atmosphere over which the convective mass flux is exactly constrained. The set of all possible states of the smaller system is termed the canonical ensemble (Chandler 1987). The temperature, $T$, of the gas is related to the mean energy per degree of freedom for the system, and is thus analogous to the mean mass flux per cloud, $\langle m\rangle$. A measure of the disorder of the system is given by the entropy, which can be defined as $S=\partial E / \partial T$. The corresponding quantity for the convective system is $\partial\langle M\rangle / \partial\langle m\rangle$, equal to $\langle M\rangle /\langle m\rangle$ if $\langle m\rangle$ is independent of the large-scale forcing. This can be understood physically by noting that $\langle N\rangle \equiv\langle M\rangle /\langle m\rangle$ is the mean number of clouds in the region. A larger entropy, or more disordered state, occurs when the mean mass flux, $\langle M\rangle$, is divided up among a large number of small clouds, rather than a small number of large clouds. Note that $\langle M\rangle$ and $\langle N\rangle$ are extensive variables, like $E$ and $S$ in the gas system, and are thus proportional to the size of the system, while $\langle m\rangle$, like the temperature of the gas, is an intensive quantity, independent of system size.

There are, however, several aspects of the ideal gas that are not reflected in the convection problem. Firstly, the number of particles in a gas is fixed, while there is no similar constraint on the number of clouds. In this sense the problem is more analogous to a photon gas than a gas composed of atoms or molecules (Chandler 1987). Furthermore for an ideal gas, energy is required to reduce the volume (pressure work). There is no analogous term in the convective problem described here. Finally, there is, of course, no quantization of convective mass flux.

\section{REFERENCES}

Arakawa, A., and W. H. Schubert, 1974: Interaction of a cumulus cloud ensemble with the large-scale environment, Part 1. J. Atmos. Sci., 31, 674-701.

Astin, I., and B. G. Latter, 1998: A case for exponential cloud fields? J. Appl. Meteor., 37, 1375-1382.
Bjerknes, J., 1938: Saturated-adiabatic ascent of air through a dry-adiabatically descending environment. Quart. J. Roy. Meteor. Soc., 64, 325-330.

Bretherton, C. S., 1988: A theory for nonprecipitating convection between two parallel plates. Part II: Nonlinear theory and cloud field organization. J. Atmos. Sci., 45, 2391-2415.

Buizza, R., M. Miller, and T. Palmer, 1999: Stochastic representation of model uncertainties in the ECMWF ensemble prediction system. Quart. J. Roy. Meteor. Soc., 125, 2887-2908.

Cahalan, R. F., and J. H. Joseph, 1989: Fractal statistics of cloud fields. Mon. Wea. Rev., 117, 261-272.

Chandler, D., 1987: Introduction to Modern Statistical Mechanics. Oxford University Press, $274 \mathrm{pp}$.

Cho, H.-R., 1978: Some statistical properties of a homogeneous and stationary shallow cumulus cloud field. J. Atmos. Sci., 35, $125-138$.

Cohen, B. G., 2001: Fluctuations in an ensemble of cumulus clouds. Ph.D. thesis, University of Reading, 165 pp.

— , and G. C. Craig, 2004: The response time of a convective cloud ensemble to a change in forcing. Quart. J. Roy. Meteor. Soc., 130, 933-944.

— and - 2006: Fluctuations in an equilibrium convective ensemble. Part II: Numerical experiments. J. Atmos. Sci., 63, 2005-2015.

Cotton, W. R., and R. A. Anthes, 1989: Storm and Cloud Dynamics. Academic Press, 883 pp.

Eagleson, P. S., 1978: Climate, soil, and vegetation. 2. The distribution of annual precipitation derived from observed storm sequences. Water Resour. Res., 14, 713-721.

Emanuel, K. A., 1991: A scheme for representing cumulus convection in large-scale models. J. Atmos. Sci., 48, 2313-2335.

Fritsch, J. M., and Coauthors, 1998: Quantitative precipitation forecasting: Report of the eighth prospectus development team, U.S. weather research program. Bull. Amer. Meteor. Soc., 79, 285-299.

Gregory, D., 1997: The mass flux approach to the parameterization of deep convection. The Physics and Parameterization of Moist Atmospheric Convection, R. K. Smith, Ed., NATO ASI Series, Vol. 505, Elsevier, 297-319.

_ - and M. J. Miller, 1989: A numerical study of the parameterization of deep tropical convection. Quart. J. Roy. Meteor. Soc., 115, 1209-1241.

Gupta, V. K., and E. C. Waymire, 1979: A stochastic kinematic study of subsynoptic space-time rainfall. Water Resour. Res., 15, 637-644.

Hozumi, K., T. Harimaya, and C. Magono, 1982: The size distribution of cumulus clouds as a function of cloud amount. $J$. Meteor. Soc. Japan, 60, 691-699.

Jorgensen, D. P., and M. A. Lemone, 1989: Vertical velocity characteristics of oceanic convection. J. Atmos. Sci., 46, 621-640.

Kavvas, M. L., and J. W. Delleur, 1981: A stochastic cluster model of daily rainfall sequences. Water Resour. Res., 17, 1151-1160.

Landau, L. D., and E. M. Lifshitz, 1968: Statistical Physics. Vol. 5. Course of Theoretical Physics, Pergamon Press, 512 pp.

Lesieur, M., 1997: Turbulence in Fluids. Kluwer Academic, 548 pp.

Lin, J. W., and J. D. Neelin, 2000: Influence of a stochastic moist convective parameterization on tropical climate variability. Geophys. Res. Lett., 27, 3691-3694.

Lopez, R. E., 1978: Internal structure and development processes of C-scale aggregates of cumulus clouds. Mon. Wea. Rev., 106, 1488-1494.

, D. O. Blanchard, D. Rosenfeld, W. L. Hiscox, and M. J. 
Casey, 1984: Population characteristics, development processes and structure of radar echoes in south Florida. Mon. Wea. Rev., 112, 56-75.

Nair, U. S., R. C. Weger, K. S. Kuo, and R. M. Welch, 1998: Clustering, randomness, and regularity in cloud fields. 5. The nature of regular cumulus cloud fields. J. Geophys. Res., 103, $11363-11380$.

Ooyama, K., 1971: A theory on parameterization of cumulus convection. J. Meteor. Soc. Japan, 49, 744-756.

Palmer, T., 2001: A nonlinear dynamical perspective on model error: A proposal for nonlocal stochastic-dynamic parameterisation in weather and climate prediction models. Quart. J. Roy. Meteor. Soc., 127, 279-304.

Plank, V. G., 1969: The size distribution of cumulus clouds in representative Florida populations. J. Appl. Meteor., 8, 46-67.

Ramirez, J. A., R. L. Bras, and K. A. Emanuel, 1990: Stabilization functions of unforced cumulus clouds: Their nature and components. J. Geophys. Res., 95, 2047-2059.

Randall, D. A., and G. J. Huffman, 1980: A stochastic model of cumulus clumping. J. Atmos. Sci., 37, 2068-2078.

Robe, F. R., and K. A. Emanuel, 1996: Moist convective scaling: Some inferences from three-dimensional cloud ensemble simulations. J. Atmos. Sci., 53, 3265-3275.

Rodriguez-Iturbe, I., D. R. Cox, and V. Isham, 1987: Some models for rainfall based on stochastic point processes. Proc. Roy. Soc. London, 410A, 269-288.

Sengupta, S. K., R. M. Welch, M. S. Navar, T. A. Berendes, and D. W. Chen, 1990: Cumulus cloud field morphology and spatial patterns derived from high spatial resolution Landsat imagery. J. Appl. Meteor., 29, 1245-1267.

Stauffer, D., and A. Ahorony, 1992: Introduction to Percolation Theory. Taylor and Francis, 190 pp.

Taylor, H. M., and S. Karlin, 1994: An Introduction to Stochastic Modelling. Academic Press, 631 pp.

Tiedke, M., 1989: A comprehensive mass flux scheme for cumulus parameterization in large-scale models. Mon. Wea. Rev., 117, 1779-1800.

Xu, K.-M., and D. A. Randall, 2001: Updraft and downdraft statistics of simulated tropical and midlatitude cumulus convection. J. Atmos. Sci., 58, 1630-1645.

— , T. Wong, B. A. Wielicki, L. Parker, and Z. A. Eitzen, 2005: Statistical analysis of satellite cloud object data from CERES. Part I: Methodology and preliminary results of the $1998 \mathrm{El}$ Niño/2000 La Niña. J. Climate, 18, 2497-2514.

Yanai, M., S. Esbenson, and J.-H. Chu, 1973: Determination of bulk properties of tropical cloud clusters from large scale heat and moisture budgets. J. Atmos. Sci., 30, 611-627. 